\title{
On flux integrals for generalized Melvin solution related to simple finite-dimensional Lie algebra
}

\author{
V. D. Ivashchuk ${ }^{1,2, a}$ \\ ${ }^{1}$ Center for Gravitation and Fundamental Metrology, VNIIMS, 46 Ozyornaya Str., Moscow 119361, Russia \\ 2 Institute of Gravitation and Cosmology, Peoples' Friendship University of Russia (RUDN University), 6 Miklukho-Maklaya Str., Moscow 117198, \\ Russia
}

Received: 27 June 2017 / Accepted: 12 September 2017 / Published online: 30 September 2017

(C) The Author(s) 2017. This article is an open access publication

\begin{abstract}
A generalized Melvin solution for an arbitrary simple finite-dimensional Lie algebra $\mathcal{G}$ is considered. The solution contains a metric, $n$ Abelian 2-forms and $n$ scalar fields, where $n$ is the rank of $\mathcal{G}$. It is governed by a set of $n$ moduli functions $H_{s}(z)$ obeying $n$ ordinary differential equations with certain boundary conditions imposed. It was conjectured earlier that these functions should be polynomialsthe so-called fluxbrane polynomials. These polynomials depend upon integration constants $q_{s}, s=1, \ldots, n$. In the case when the conjecture on the polynomial structure for the Lie algebra $\mathcal{G}$ is satisfied, it is proved that 2-form flux integrals $\Phi^{s}$ over a proper $2 d$ submanifold are finite and obey the relations $q_{s} \Phi^{s}=4 \pi n_{s} h_{s}$, where the $h_{s}>0$ are certain constants (related to dilatonic coupling vectors) and the $n_{s}$ are powers of the polynomials, which are components of a twice dual Weyl vector in the basis of simple (co-)roots, $s=1, \ldots, n$. The main relations of the paper are valid for a solution corresponding to a finite-dimensional semi-simple Lie algebra $\mathcal{G}$. Examples of polynomials and fluxes for the Lie algebras $A_{1}, A_{2}, A_{3}, C_{2}, G_{2}$ and $A_{1}+A_{1}$ are presented.
\end{abstract}

\section{Introduction}

In this paper we start with a generalization of a Melvin solution [1], which was presented earlier in Ref. [2]. It appears in the model which contains a metric, $n$ Abelian 2-forms and $l \geq n$ scalar fields. This solution is governed by a certain nondegenerate (quasi-Cartan) matrix $\left(A_{s s^{\prime}}\right), s, s^{\prime}=1, \ldots, n$. It is a special case of the so-called generalized fluxbrane solutions from Ref. [3]. For fluxbrane solutions see Refs. [4-28] and the references therein. The appearance of fluxbrane solutions was motivated by superstring/ $M$ theory.

\footnotetext{
a e-mail: ivashchuk@mail.ru
}

The generalized fluxbrane solutions from Ref. [3] are governed by moduli functions, $H_{S}(z)>0$, defined on the interval $(0,+\infty)$, where $z=\rho^{2}$ and $\rho$ is a radial variable. These functions obey a set of $n$ non-linear differential master equations governed by the matrix $\left(A_{s s^{\prime}}\right)$, equivalent to Toda-like equations, with the following boundary conditions imposed: $H_{s}(+0)=1, s=1, \ldots, n$.

In this paper we assume that $\left(A_{s s^{\prime}}\right)$ is a Cartan matrix for some simple finite-dimensional Lie algebra $\mathcal{G}$ of rank $n$ $\left(A_{s s}=2\right.$ for all $\left.s\right)$. According to a conjecture suggested in Ref. [3], the solutions to the master equations with the boundary conditions imposed are polynomials:

$H_{S}(z)=1+\sum_{k=1}^{n_{s}} P_{s}^{(k)} z^{k}$

where the $P_{s}^{(k)}$ are constants. Here $P_{s}^{\left(n_{s}\right)} \neq 0$ and

$n_{s}=2 \sum_{s^{\prime}=1}^{n} A^{s s^{\prime}}$,

where we denote $\left(A^{s s^{\prime}}\right)=\left(A_{s s^{\prime}}\right)^{-1}$. The integers $n_{s}$ are components of a twice dual Weyl vector in the basis of simple (co-)roots [29].

The set of fluxbrane polynomials $H_{S}$ defines a special solution to open Toda chain equations [30,31] corresponding to a simple finite-dimensional Lie algebra $\mathcal{G}$ [32]. In Refs. [2,33] a program (in Maple) for the calculation of these polynomials for the classical series of Lie algebras $(A-, B-, C$ - and $D$-series) was suggested. It was pointed out in Ref. [3] that the conjecture on the polynomial structure of $H_{S}(z)$ is valid for Lie algebras of the $A$ - and $C$-series. In Ref. [34] the conjecture from Ref. [3] was verified for the Lie algebra $E_{6}$ and certain duality relations for six $E_{6}$-polynomials were proved. In Sect. 2 we present the generalized Melvin solution from 
Ref. [2]. In Sect. 3 we deal with the generalized Melvin solution for an arbitrary simple finite-dimensional Lie algebra $\mathcal{G}$. Here we calculate 2-form flux integrals $\Phi^{s}=\int_{M_{*}} F^{s}$, where $F^{s}$ are 2-forms and $M_{*}$ is a certain $2 d$ submanifold. These integrals (fluxes) are finite when moduli functions are polynomials. In Sect. 3 we consider examples of fluxbrane polynomials and fluxes for the Lie algebras: $A_{1}, A_{2}, A_{3}, C_{2}$, $G_{2}$ and $A_{1}+A_{1}$.

\section{The solutions}

We consider a model governed by the action

$$
\begin{aligned}
S= & \int d^{D} x \sqrt{|g|}\left\{R[g]-h_{\alpha \beta} g^{M N} \partial_{M} \varphi^{\alpha} \partial_{N} \varphi^{\beta}\right. \\
& \left.-\frac{1}{2} \sum_{s=1}^{n} \exp \left[2 \lambda_{s}(\varphi)\right]\left(F^{s}\right)^{2}\right\}
\end{aligned}
$$

where $g=g_{M N}(x) \mathrm{d} x^{M} \otimes \mathrm{d} x^{N}$ is a metric, $\varphi=\left(\varphi^{\alpha}\right) \in \mathbb{R}^{l}$ is a set of scalar fields, $\left(h_{\alpha \beta}\right)$ is a constant symmetric nondegenerate $l \times l$ matrix $(l \in \mathbb{N}), F^{s}=d A^{s}=\frac{1}{2} F_{M N}^{s} \mathrm{~d} x^{M} \wedge$ $\mathrm{d} x^{N}$ is a 2-form, $\lambda_{s}$ is a 1-form on $\mathbb{R}^{l}: \lambda_{s}(\varphi)=\lambda_{s \alpha} \varphi^{\alpha}, s=$ $1, \ldots, n ; \alpha=1, \ldots, l$. Here $\left(\lambda_{s \alpha}\right), s=1, \ldots, n$, are dilatonic coupling vectors. In (2.1) we denote $|g|=\left|\operatorname{det}\left(g_{M N}\right)\right|$, $\left(F^{s}\right)^{2}=F_{M_{1} M_{2}}^{s} F_{N_{1} N_{2}}^{s} g^{M_{1} N_{1}} g^{M_{2} N_{2}}, s=1, \ldots, n$.

Here we start with a family of exact solutions to field equations corresponding to the action (2.1) and depending on one variable $\rho$. The solutions are defined on the manifold

$M=(0,+\infty) \times M_{1} \times M_{2}$,

where $M_{1}$ is a one-dimensional manifold (say $S^{1}$ or $\mathbb{R}$ ) and $M_{2}$ is a (D-2)-dimensional Ricci-flat manifold. The solution reads [2]

$$
\begin{aligned}
g= & \left(\prod_{s=1}^{n} H_{s}^{2 h_{s} /(D-2)}\right)\{w \mathrm{~d} \rho \otimes \mathrm{d} \rho \\
& \left.+\left(\prod_{s=1}^{n} H_{s}^{-2 h_{s}}\right) \rho^{2} \mathrm{~d} \phi \otimes \mathrm{d} \phi+g^{2}\right\}, \\
\exp \left(\varphi^{\alpha}\right)= & \prod_{s=1}^{n} H_{s}^{h_{s} \lambda_{s}^{\alpha}}, \\
F^{s}= & q_{s}\left(\prod_{s^{\prime}=1}^{n} H_{s^{\prime}}^{-A_{s s^{\prime}}}\right) \rho \mathrm{d} \rho \wedge \mathrm{d} \phi,
\end{aligned}
$$

$s=1, \ldots, n ; \alpha=1, \ldots, l$, where $w= \pm 1, g^{1}=\mathrm{d} \phi \otimes \mathrm{d} \phi$ is a metric on $M_{1}$ and $g^{2}$ is a Ricci-flat metric on $M_{2}$. Here $q_{s} \neq 0$ are integration constants, $q_{s}=-Q_{s}$ in the notations of Ref. [2], $s=1, \ldots, n$.

The functions $H_{S}(z)>0, z=\rho^{2}$, obey the master equations $\frac{\mathrm{d}}{\mathrm{d} z}\left(\frac{z}{H_{S}} \frac{\mathrm{d}}{\mathrm{d} z} H_{s}\right)=P_{s} \prod_{s^{\prime}=1}^{n} H_{s^{\prime}}^{-A_{s s^{\prime}}}$,

with the following boundary conditions:

$H_{S}(+0)=1$,

where

$P_{s}=\frac{1}{4} K_{s} q_{s}^{2}$

$s=1, \ldots, n$. The boundary condition (2.7) guarantees the absence of a conic singularity [in the metric (2.3)] for $\rho=$ +0 .

The parameters $h_{s}$ satisfy the relations

$h_{s}=K_{s}^{-1}, \quad K_{s}=B_{s s}>0$,

where

$B_{s s^{\prime}} \equiv 1+\frac{1}{2-D}+\lambda_{s \alpha} \lambda_{s^{\prime} \beta} h^{\alpha \beta}$

$s, s^{\prime}=1, \ldots, n$, with $\left(h^{\alpha \beta}\right)=\left(h_{\alpha \beta}\right)^{-1}$. In the relations above we denote $\lambda_{s}^{\alpha}=h^{\alpha \beta} \lambda_{s \beta}$ and

$\left(A_{s s^{\prime}}\right)=\left(2 B_{s s^{\prime}} / B_{s^{\prime} s^{\prime}}\right)$

The latter is the so-called quasi-Cartan matrix.

We note that the constants $B_{s s^{\prime}}$ and $K_{s}=B_{s s}$ have a certain mathematical sense. They are related to scalar products of certain vectors $U^{s}$ (brane vectors, or $U$-vectors), which belong to a certain linear space ("truncated target space", for our problem it has dimension $l+2)$, i.e. $B_{s s^{\prime}}=\left(U^{s}, U^{s^{\prime}}\right)$ and $K_{s}=\left(U^{s}, U^{s}\right)$ [35-37]. The scalar products of such a type are of physical significance, since they appear for various solutions with branes, e.g. black branes, $S$-branes, fluxbranes etc. Several physical parameters in multidimensional models with branes, e.g. the Hawking-like temperatures and the entropies of black holes and branes, PPN parameters, Hubble-like parameters, fluxes etc., contain such scalar products; see [36,37] and Sect. 3 of this paper. The relation (2.11) defines generalized intersection rules for branes which were suggested in [35]. The constants $K_{S}$ are invariants of dimensional reduction. It is well known, see [37] and the references therein, that $K_{s}=2$ for branes in numerous supergravity models, e.g. in dimensions $D=10,11$.

It may be shown that if the matrix $\left(h_{\alpha \beta}\right)$ has an Euclidean signature and $l \geq n$, and $\left(A_{s s^{\prime}}\right)$ is a Cartan matrix for a simple Lie algebra $\mathcal{G}$ of $\operatorname{rank} n$, there exists a set of co-vectors $\lambda_{1}, \ldots, \lambda_{n}$ obeying (2.11) (for $l=n$ see Remark 1 in the next section). Thus the solution is valid at least when $l \geq n$ and the matrix $\left(h_{\alpha \beta}\right)$ is positive-definite. 
The solution under consideration is a special case of the fluxbrane (for $\left.w=+1, M_{1}=S^{1}\right)$ and $S$-brane $(w=-1$ ) solutions from [3] and [25], respectively.

If $w=+1$ and the (Ricci-flat) metric $g^{2}$ has a pseudoEuclidean signature, we get a multidimensional generalization of Melvin's solution [1].

In our notations Melvin's solution (without scalar field) corresponds to $D=4, n=1, l=0, M_{1}=S^{1}(0<\phi<$ $2 \pi), M_{2}=\mathbb{R}^{2}, g^{2}=-\mathrm{d} t \otimes \mathrm{d} t+\mathrm{d} x \otimes \mathrm{d} x$ and $\mathcal{G}=A_{1}$.

For $w=-1$ and $g^{2}$ of Euclidean signature we obtain a cosmological solution with a horizon (as $\rho=+0)$ if $M_{1}=\mathbb{R}$ $(-\infty<\phi<+\infty)$.

\section{Flux integrals for a simple finite-dimensional Lie algebra}

Here we deal with the solution which corresponds to a simple finite-dimensional Lie algebra $\mathcal{G}$, i.e. the matrix $A=\left(A_{s s^{\prime}}\right)$ is coinciding with the Cartan matrix of this Lie algebra. We put also $n=l, w=+1$ and $M_{1}=S^{1}, h_{\alpha \beta}=\delta_{\alpha \beta}$ and denote $\left(\lambda_{s a}\right)=\left(\lambda_{s}^{a}\right)=\lambda_{s}, s=1, \ldots, n$.

Due to (2.9)-(2.11) we get

$K_{s}=\frac{D-3}{D-2}+\lambda_{s}^{2}$,

$h_{s}=K_{s}^{-1}$, and

$\lambda_{s} \lambda_{l}=\frac{1}{2} K_{l} A_{s l}-\frac{D-3}{D-2} \equiv \Gamma_{s l}$,

$s, l=1, \ldots, n$. [Equation (3.1) is a special case of (3.2)].

It follows from (2.9)-(2.11) that

$\frac{h_{i}}{h_{j}}=\frac{K_{j}}{K_{i}}=\frac{B_{j j}}{B_{i i}}=\frac{B_{j i}}{B_{i i}} \frac{B_{j j}}{B_{i j}}=\frac{A_{j i}}{A_{i j}}$

for any $i \neq j$ obeying $A_{i j}, A_{j i} \neq 0 ; i, j=1, \ldots, n$. It may be readily shown from (3.3) that the ratios $\frac{h_{i}}{h_{j}}=\frac{K_{j}}{K_{i}}$ are fixed numbers for any given Cartan matrix $\left(A_{i j}\right)$ of a simple (finite-dimensional) Lie algebra $\mathcal{G}$. (This follows from (3.3) and the connectedness of the Dynkin diagram of a simple Lie algebra.) The ratios (3.3) may be written as follows:

$\frac{h_{i}}{h_{j}}=\frac{K_{j}}{K_{i}}=\frac{r_{j}}{r_{i}}$

$i \neq j$, where $r_{i}=\left(\alpha_{i}, \alpha_{i}\right)$ is the length squared of a simple root $\alpha_{i}$ corresponding to the Lie algebra $\mathcal{G}$. Here we use the notations $A_{i j}=2\left(\alpha_{i}, \alpha_{j}\right) /\left(\alpha_{j}, \alpha_{j}\right) ; i, j=1, \ldots, n$. Equation (3.4) implies

$K_{i}=\frac{1}{2} K r_{i}$ $i=1, \ldots, n$, where $K>0$. (For simply laced $(A, D, E)$ Lie algebras all $r_{i}$ are equal.)

Remark 1 For large enough $K$ in (3.5) there exist vectors $\lambda_{s}$ obeying (3.2) [and hence (3.1)]. Indeed, the matrix $\left(\Gamma_{s l}\right)$ is positive-definite if $K>K_{*}$, where $K_{*}$ is some positive number. Hence there exists a matrix $\Lambda$, such that $\Lambda^{T} \Lambda=\Gamma$. We put $\left(\Lambda_{a s}\right)=\left(\lambda_{s}^{a}\right)$ and get the set of vectors obeying (3.2).

Now let us consider the oriented 2-dimensional manifold $M_{*}=(0,+\infty) \times S^{1}$. The flux integrals

$$
\begin{aligned}
\Phi^{s} & =\int_{M_{*}} F^{s}=\int_{0}^{+\infty} \mathrm{d} \rho \int_{0}^{2 \pi} \mathrm{d} \phi \rho \mathcal{B}^{s}\left(\rho^{2}\right) \\
& =2 \pi \int_{0}^{+\infty} \mathrm{d} \rho \rho \mathcal{B}^{s}\left(\rho^{2}\right),
\end{aligned}
$$

where

$\mathcal{B}^{s}\left(\rho^{2}\right)=q_{s} \prod_{l=1}^{n}\left(H_{l}\left(\rho^{2}\right)\right)^{-A_{s l}}$,

are convergent for all $s$, if the conjecture for the Lie algebra $\mathcal{G}$ (on polynomial structure of moduli functions $H_{S}$ ) is obeyed for the Lie algebra $\mathcal{G}$ under consideration.

Indeed, due to the polynomial assumption (1.1) we have

$H_{s}\left(\rho^{2}\right) \sim C_{s} \rho^{2 n_{s}}, \quad C_{s}=P_{s}^{\left(n_{s}\right)}$,

as $\rho \rightarrow+\infty ; s=1, \ldots, n$. From (3.7), (3.8) and the equality $\sum_{1}^{n} A_{s l} n_{l}=2$, following from (1.2), we get

$\mathcal{B}^{s}\left(\rho^{2}\right) \sim q_{s} C^{s} \rho^{-4}, \quad C^{s}=\prod_{l=1}^{n} C_{l}^{-A_{s l}}$,

and hence the integral (3.6) is convergent for any $s=$ $1, \ldots, n$.

By using the master equations (2.6) we obtain

$$
\begin{aligned}
\int_{0}^{+\infty} \mathrm{d} \rho \rho \mathcal{B}^{s}\left(\rho^{2}\right) & =q_{s} P_{s}^{-1} \frac{1}{2} \int_{0}^{+\infty} \mathrm{d} z \frac{\mathrm{d}}{\mathrm{d} z}\left(\frac{z}{H_{s}} \frac{\mathrm{d}}{\mathrm{d} z} H_{s}\right) \\
& =\frac{1}{2} q_{s} P_{s}^{-1} \lim _{z \rightarrow+\infty}\left(\frac{z}{H_{s}} \frac{\mathrm{d}}{\mathrm{d} z} H_{s}\right) \\
& =\frac{1}{2} n_{s} q_{s} P_{s}^{-1}
\end{aligned}
$$

which implies [see (2.8)]

$\Phi^{s}=4 \pi n_{s} q_{s}^{-1} h_{s}$,

$s=1, \ldots, n$.

Thus, any flux $\Phi^{s}$ depends upon one integration constant $q_{s} \neq 0$, while the integrand form $F^{s}$ depends upon all constants: $q_{1}, \ldots, q_{n}$. 
We note that for $D=4$ and $g^{2}=-\mathrm{d} t \otimes \mathrm{d} t+\mathrm{d} x \otimes d x$, $q_{s}$ is coinciding with the value of the $x$-component of the $s$ th magnetic field on the axis of symmetry.

In the case of the Gibbons-Maeda dilatonic generalization of the Melvin solution, corresponding to $D=4, n=l=1$ and $\mathcal{G}=A_{1}$ [5], the flux from (3.11) $(s=1)$ is in agreement with that considered in Ref. [26]. For Melvin's case and some higher dimensional extensions (with $\mathcal{G}=A_{1}$ ) see also Ref. [14].

Due to (3.4) the ratios

$\frac{q_{i} \Phi^{i}}{q_{j} \Phi^{j}}=\frac{n_{i} h_{i}}{n_{j} h_{j}}=\frac{n_{i} r_{j}}{n_{j} r_{i}}$

are fixed numbers depending upon the Cartan matrix $\left(A_{i j}\right)$ of a simple finite-dimensional Lie algebra $\mathcal{G}$.

Remark 2 The relation for flux integrals (3.11) is also valid when the matrix $\left(A_{s s^{\prime}}\right)$ is a Cartan matrix of a finitedimensional semi-simple Lie algebra $\mathcal{G}=\mathcal{G}_{1} \oplus \cdots \oplus \mathcal{G}_{k}$, where $\mathcal{G}_{1}, \ldots, \mathcal{G}_{k}$ are simple Lie (sub)algebras. In this case the Cartan matrix $\left(A_{i j}\right)$ has a block-diagonal form, i.e. $\left(A_{i j}\right)=\operatorname{diag}\left(\left(A_{i_{1} j_{1}}^{(1)}\right), \ldots,\left(A_{i_{k} j_{k}}^{(k)}\right)\right)$, where $\left(A_{i_{a} j_{a}}^{(a)}\right)$ is the Cartan matrix of the Lie algebra $\mathcal{G}_{a}, a=1, \ldots, k$. The set of polynomials in this case splits in a direct union of sets of polynomials corresponding to the Lie algebras $\mathcal{G}_{1}, \ldots, \mathcal{G}_{k}$. Equations (3.4) and (3.12) are valid, when the indices $i, j$ correspond to one $a$ th block, $a=1, \ldots, k$. The quantities $q_{i} \Phi^{i}$ and $q_{j} \Phi^{j}$ corresponding to different blocks are independent. Equation (3.5) should be replaced by

$K_{i_{a}}=\frac{1}{2} K^{(a)} r_{i_{a}}, \quad K^{(a)}>0$,

for any index $i_{a}$ corresponding to the $a$ th block; $a=1, \ldots, k$. The existence of dilatonic coupling vectors $\lambda_{s}$ obeying (3.2) [(and (3.1)] just follows from the arguments of Remark 1, if we put all $K^{(a)}=K>0$.

The manifold $M_{*}=(0,+\infty) \times S^{1}$ is isomorphic to the manifold $\mathbb{R}_{*}^{2}=\mathbb{R}^{2} \backslash\{0\}$. The solution (2.3)-(2.5) may be understood (or rewritten by pull-backs) as defined on the manifold $\mathbb{R}_{*}^{2} \times M_{2}$, where the coordinates $\rho, \phi$ are understood as coordinates on $\mathbb{R}_{*}^{2}$. They are not globally defined. One should consider two charts with coordinates $\rho, \phi=\phi_{1}$ and $\rho, \phi=\phi_{2}$, where $\rho>0,0<\phi_{1}<2 \pi$ and $-\pi<\phi_{2}<$ $\pi$. Here $\exp \left(i \phi_{1}\right)=\exp \left(i \phi_{2}\right)$. In both cases we have $x=$ $\rho \cos \phi$ and $y=\rho \sin \phi$, where $x, y$ are standard coordinates of $\mathbb{R}^{2}$. Using the identity $\rho \mathrm{d} \rho \wedge \mathrm{d} \phi=\mathrm{d} x \wedge \mathrm{d} y$ we get

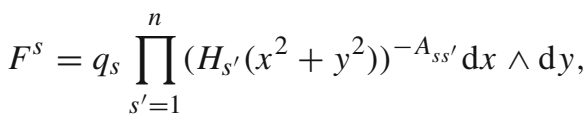

$s=1, \ldots, n$. The 2-forms (3.14) are well defined on $\mathbb{R}^{2}$. Indeed, due to the conjecture from Ref. [3] any polynomial $H_{s}(z)$ is a smooth function on $\mathbb{R}=(-\infty,+\infty)$ which obeys $H_{S}(z)>0$ for $z \in\left(-\varepsilon_{s},+\infty\right)$, where $\varepsilon_{s}>0$. This is valid due to the conjecture from Ref. [3] $H_{S}(z)>0$ for $z>0$ and $H_{s}(+0)=1$. Thus, $\left(\prod_{s^{\prime}=1}^{n}\left(H_{s^{\prime}}\left(x^{2}+y^{2}\right)\right)^{-A_{s s^{\prime}}}\right)$ is a smooth function since it is a composition of two well-defined smooth functions $\left(\prod_{s^{\prime}=1}^{n}\left(H_{s^{\prime}}(z)\right)^{-A_{s s^{\prime}}}\right)$ and $z=x^{2}+y^{2}$.

Now we show that there exist 1 -forms $A^{s}$ obeying $F^{s}=$ $d A^{s}$ which are globally defined on $\mathbb{R}^{2}$. We start with the open submanifold $\mathbb{R}_{*}^{2}$. The 1-forms

$A^{s}=\left(\int_{0}^{\rho} \mathrm{d} \bar{\rho} \bar{\rho} \mathcal{B}^{s}\left(\bar{\rho}^{2}\right)\right) \mathrm{d} \phi=\frac{1}{2}\left(\int_{0}^{\rho^{2}} \mathrm{~d} \bar{z} \mathcal{B}^{s}(\bar{z})\right) \mathrm{d} \phi$

are well defined on $\mathbb{R}_{*}^{2}$ (here $\mathrm{d} \phi=\left(x^{2}+y^{2}\right)^{-1}(-y \mathrm{~d} x+$ $x \mathrm{~d} y)$ ) and obey $F^{s}=d A^{s}, s=1, \ldots, n$. Using the master equation (2.6) we obtain

$$
\begin{aligned}
A^{s} & =\frac{q_{s}}{2 P_{s}}\left(\int_{0}^{\rho^{2}} \mathrm{~d} \bar{z} \frac{\mathrm{d}}{\mathrm{d} \bar{z}}\left(\frac{\bar{z}}{H_{S}(\bar{z})} \frac{\mathrm{d}}{\mathrm{d} \bar{z}} H_{S}(\bar{z})\right)\right) \mathrm{d} \phi \\
& =\frac{2 h_{s}}{q_{s}} \frac{H_{S}^{\prime}\left(\rho^{2}\right)}{H_{S}\left(\rho^{2}\right)} \rho^{2} d \phi,
\end{aligned}
$$

$s=1, \ldots, n$. Here $H^{\prime}{ }_{s}=\frac{\mathrm{d}}{\mathrm{d} z} H_{s}$. Due to the relation $\rho^{2} \mathrm{~d} \phi=$ $-y \mathrm{~d} x+x \mathrm{~d} y$, we obtain

$A^{s}=\frac{2 h_{s}}{q_{s}} \frac{H_{s}^{\prime}\left(x^{2}+y^{2}\right)}{H_{s}\left(x^{2}+y^{2}\right)}(-y \mathrm{~d} x+x \mathrm{~d} y)$,

$s=1, \ldots, n$. The 1 -forms (3.17) are well-defined smooth 1 -forms on $\mathbb{R}^{2}$.

We note that in the case of the Gibbons-Maeda solution [5] corresponding to $D=4, n=l=1$ and $\mathcal{G}=A_{1}$ the gauge potential from (3.16) coincides (up to notations) with that considered in Ref. [7].

Now we verify our result (3.11) for flux integrals by using the relations for the 1 -forms $A^{s}$. Let us consider a $2 d$ oriented manifold (disk) $D_{R}=\left\{(x, y): x^{2}+y^{2} \leq R^{2}\right\}$ with the boundary $\partial D_{R}=C_{R}=\left\{(x, y): x^{2}+y^{2}=R^{2}\right\} . C_{R}$ is a circle of radius $R$. It is an $1 d$ oriented manifold with the orientation (inherited from that of $D_{R}$ ) obeying the relation $\int_{C_{R}} \mathrm{~d} \phi=2 \pi$. Using the Stokes-Cartan theorem we get

$\int_{D_{R}} F^{s}=\int_{D_{R}} \mathrm{~d} A^{s}=\int_{C_{R}} A^{s}=\frac{4 \pi h_{s}}{q_{s}} \frac{H_{s}^{\prime}\left(R^{2}\right)}{H_{s}\left(R^{2}\right)} R^{2}$,

$s=1, \ldots, n$. By using the asymptotic relation (3.8) we find

$\lim _{R \rightarrow+\infty} \int_{D_{R}} F^{s}=\frac{4 \pi h_{s} n_{s}}{q_{s}}$

$s=1, \ldots, n$, in agreement with (3.11). 
Remark 3 We note (for completeness) that the metric and scalar fields for our solution with $w=+1$ and $l=n$ can be extended to the manifold $\mathbb{R}^{2} \times M_{2}$. Indeed, in the coordinates $x, y$ the metric (2.3) and scalar fields (2.4) read as follows:

$$
\begin{aligned}
g= & \left(\prod_{s=1}^{n} H_{s}^{2 h_{s} /(D-2)}\right)\{\mathrm{d} x \otimes \mathrm{d} x+\mathrm{d} y \otimes \mathrm{d} y \\
& \left.+f(-y \mathrm{~d} x+x \mathrm{~d} y)^{2}+g^{2}\right\},
\end{aligned}
$$

$\varphi^{a}=\sum_{s=1}^{n} h_{s} \lambda_{s}^{a} \ln H_{s}$,

$a=1, \ldots, l$. Here $H_{s}=H_{s}\left(x^{2}+y^{2}\right), s=1, \ldots, n$, and $f=f\left(x^{2}+y^{2}\right)$, where

$f(z)=\left(\left(\prod_{s=1}^{n}\left(H_{S}(z)\right)^{-2 h_{s}}\right)-1\right) z^{-1}$,

for $z \neq 0$ and $f(0)=\lim _{z \rightarrow 0} f(z)$ (the limit does exist). The function $f(z)$ is smooth in the interval $(-\varepsilon,+\infty)$ for some $\varepsilon>0$. Indeed, it is smooth in the interval $(0,+\infty)$ and holomorphic in the domain $\{z|0<| z \mid<\varepsilon\}$ for a small

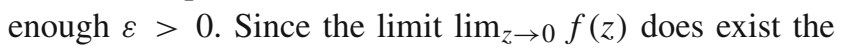
function $f(z)$ is holomorphic in the disc $\{z|| z \mid<\varepsilon\}$ and hence it is smooth in the interval $(-\varepsilon,+\infty)$. This implies that the metric is smooth on the manifold $\mathbb{R}^{2} \times M_{2}$. (See the text after Eq. (3.14).) The scalar fields are also smooth on $\mathbb{R}^{2} \times M_{2}$.

\section{Examples}

Here we present fluxbrane polynomials corresponding to the Lie algebras $A_{1}, A_{2}, A_{3}, C_{2}, G_{2}, A_{1}+A_{1}$ and related fluxes. Here as in [32] we use other parameters $p_{s}$ instead of $P_{s}$ :

$p_{s}=P_{s} / n_{s}$,

$s=1, \ldots, n$.

$A_{1}$-case. The simplest example occurs in the case of the Lie algebra $A_{1}=\operatorname{sl}(2)$. Here $n_{1}=1$. We get [3]

$H_{1}=1+p_{1} z$

and

$\Phi^{1}=4 \pi q_{1}^{-1} h_{1}$,

which is also valid for Melvin's solution with $D=4$ and $h_{1}=2$.

$A_{2}$-case. For the Lie algebra $A_{2}=s l(3)$ with the Cartan matrix

$\left(A_{s s^{\prime}}\right)=\left(\begin{array}{cc}2 & -1 \\ -1 & 2\end{array}\right)$ we have $[3,25,32] n_{1}=n_{2}=2$ and

$H_{1}=1+2 p_{1} z+p_{1} p_{2} z^{2}$,

$H_{2}=1+2 p_{2} z+p_{1} p_{2} z^{2}$.

We get in this case

$\left(\Phi^{1}, \Phi^{2}\right)=8 \pi h\left(q_{1}^{-1}, q_{2}^{-1}\right)$,

where $h_{1}=h_{2}=h$.

$A_{3}$-case. The polynomials for the $A_{3}$-case read as follows [32,33]:

$\begin{aligned} H_{1}= & 1+3 p_{1} z+3 p_{1} p_{2} z^{2}+p_{1} p_{2} p_{3} z^{3} \\ H_{2}= & 1+4 p_{2} z+3\left(p_{1} p_{2}+p_{2} p_{3}\right) z^{2} \\ & +4 p_{1} p_{2} p_{3} z^{3}+p_{1} p_{2}^{2} p_{3} z^{4} \\ H_{3}= & 1+3 p_{3} z+3 p_{2} p_{3} z^{2}+p_{1} p_{2} p_{3} z^{3} .\end{aligned}$

Here we have $\left(n_{1}, n_{2}, n_{3}\right)=(3,4,3)$ and

$\left(\Phi^{1}, \Phi^{2}, \Phi^{3}\right)=4 \pi h\left(3 q_{1}^{-1}, 4 q_{2}^{-1}, 3 q_{3}^{-1}\right)$

with $h_{1}=h_{2}=h_{3}=h$.

$C_{2}$-case. For the Lie algebra $C_{2}=s o(5)$ with the Cartan matrix

$\left(A_{s s^{\prime}}\right)=\left(\begin{array}{cc}2 & -1 \\ -2 & 2\end{array}\right)$

we get $n_{1}=3$ and $n_{2}=4$. For $C_{2}$-polynomials we obtain $[25,32]$

$H_{1}=1+3 p_{1} z+3 p_{1} p_{2} z^{2}+p_{1}^{2} p_{2} z^{3}$,

$H_{2}=1+4 p_{2} z+6 p_{1} p_{2} z^{2}+4 p_{1}^{2} p_{2} z^{3}+p_{1}^{2} p_{2}^{2} z^{4}$.

In this case we find

$\left(\Phi^{1}, \Phi^{2}\right)=4 \pi\left(3 h_{1} q_{1}^{-1}, 4 h_{2} q_{2}^{-1}\right)$

where $h_{1}=2 h_{2}$.

$G_{2}$-case. For the Lie algebra $G_{2}$ with the Cartan matrix

$\left(A_{s s^{\prime}}\right)=\left(\begin{array}{cc}2 & -1 \\ -3 & 2\end{array}\right)$ 
we get $n_{1}=6$ and $n_{2}=10$. In this case the fluxbrane polynomials read $[25,32]$

$$
\begin{aligned}
H_{1}= & 1+6 p_{1} z+15 p_{1} p_{2} z^{2}+20 p_{1}^{2} p_{2} z^{3} \\
& +15 p_{1}^{3} p_{2} z^{4}+6 p_{1}^{3} p_{2}^{2} z^{5}+p_{1}^{4} p_{2}^{2} z^{6}, \\
H_{2}= & 1+10 p_{2} z+45 p_{1} p_{2} z^{2}+120 p_{1}^{2} p_{2} z^{3} \\
& +p_{1}^{2} p_{2}\left(135 p_{1}+75 p_{2}\right) z^{4} \\
& +252 p_{1}^{3} p_{2}^{2} z^{5}+p_{1}^{3} p_{2}^{2}\left(75 p_{1}+135 p_{2}\right) z^{6} \\
& +120 p_{1}^{4} p_{2}^{3} z^{7} \\
& +45 p_{1}^{5} p_{2}^{3} z^{8}+10 p_{1}^{6} p_{2}^{3} z^{9}+p_{1}^{6} p_{2}^{4} z^{10} .
\end{aligned}
$$

We are led to the relations

$$
\left(\Phi^{1}, \Phi^{2}\right)=4 \pi\left(6 h_{1} q_{1}^{-1}, 10 h_{2} q_{2}^{-1}\right)
$$

where $h_{1}=3 h_{2}$.

$\left(A_{1}+A_{1}\right)$-case. For the semi-simple Lie algebra $A_{1}+A_{1}$ we obtain $n_{1}=n_{2}=1$,

$H_{1}=1+p_{1} z, \quad H_{2}=1+p_{2} z$,

and

$\left(\Phi^{1}, \Phi^{2}\right)=4 \pi\left(q_{1}^{-1} h_{1}, q_{2}^{-1} h_{2}\right)$,

where $h_{1}$ and $h_{2}$ are independent, as well as the quantities $q_{1} \Phi^{1}$ and $q_{2} \Phi^{2}$.

\section{Conclusions}

Here we have considered a multidimensional generalization of Melvin's solution corresponding to a simple finitedimensional Lie algebra $\mathcal{G}$. We have assumed that the solution is governed by a set of $n$ fluxbrane polynomials $H_{S}(z)$, $s=1, \ldots, n$. These polynomials define special solutions to open Toda chain equations corresponding to the Lie algebra $\mathcal{G}$.

The polynomials $H_{s}(z)$ depend also upon parameters $q_{s}$, which are coinciding for $D=4$ (up to a sign) with the values of colored magnetic fields on the axis of symmetry.

We have calculated $2 d$ flux integrals $\Phi^{s}=\int F^{s}, s=$ $1, \ldots, n$. Any flux $\Phi^{s}$ depends only upon one parameter $q_{s}$, while the integrand $F^{s}$ depends upon all parameters $q_{1}, \ldots, q_{n}$. The relation for flux integrals (3.11) is also valid when the matrix $\left(A_{s s^{\prime}}\right)$ is a Cartan matrix of a finitedimensional semi-simple Lie algebra $\mathcal{G}$.

Here we have considered examples of polynomials and fluxes for the Lie algebras $A_{1}, A_{2}, A_{3}, C_{2}, G_{2}$ and $A_{1}+A_{1}$. The approach of this paper will be used for a calculation of certain flux integrals for forms $F^{s}$ of arbitrary ranks corresponding to certain fluxbrane solutions (of electric type by $p$-brane notation or magnetic type by fluxbrane classification $^{1}$ ) governed by fluxbrane polynomials [38].

An open problem is to find the fluxes for the solutions which are related to infinite-dimensional Lorentzian KacMoody algebras, e.g. hyperbolic ones $[39,40]$. In this case one should deal with phantom scalar fields in the model (2.1) and non-polynomial solutions to Eqs. (2.6). Another possibility is to study the convergence of flux integrals for nonpolynomial solutions for moduli functions corresponding to non-Cartan matrices $\left(A_{s s^{\prime}}\right)$ (e.g. for the model with two 2forms from Ref. [41]).

Acknowledgements This work was supported in part by the Russian Foundation for Basic Research Grant No. 16-02-00602 and by the Ministry of Education of the Russian Federation (the Agreement Number 02.a03.21.0008 of 24 June 2016).

Open Access This article is distributed under the terms of the Creative Commons Attribution 4.0 International License (http://creativecomm ons.org/licenses/by/4.0/), which permits unrestricted use, distribution, and reproduction in any medium, provided you give appropriate credit to the original author(s) and the source, provide a link to the Creative Commons license, and indicate if changes were made.

Funded by SCOAP . $^{3}$

\section{References}

1. M.A. Melvin, Pure magnetic and electric geons. Phys. Lett. 8, 65 (1964)

2. A.A. Golubtsova, V.D. Ivashchuk, On multidimensional analogs of Melvin's solution for classical series of Lie algebras. Grav. Cosmol. 15(2), 144-147 (2009). arXiv:1009.3667

3. V.D. Ivashchuk, Composite fluxbranes with general intersections. Class. Quantum Grav. 19, 3033-3048 (2002). arXiv:hep-th/0202022

4. G.W. Gibbons, D.L. Wiltshire, Spacetime as a membrane in higher dimensions. Nucl. Phys. B 287, 717-742 (1987). arXiv:hep-th/0109093

5. G. Gibbons, K. Maeda, Black holes and membranes in higher dimensional theories with dilaton fields. Nucl. Phys. B 298, 741775 (1988)

6. F. Dowker, J.P. Gauntlett, D.A. Kastor, J. Traschen, Pair creation of dilaton black holes. Phys. Rev. D 49, 2909-2917 (1994). arXiv:hep-th/9309075

7. F. Dowker, J.P. Gauntlett, S.B. Giddings, G.T. Horowitz, On pair creation of extremal black holes and Kaluza-Klein monopoles. Phys. Rev. D 50, 2662 (1994). arXiv:hep-th/9312172

8. F. Dowker, J.P. Gauntlett, G.W. Gibbons, G.T. Horowitz, The decay of magnetic fields in Kaluza-Klein theory. Phys. Rev. D 52, 6929 (1995). arXiv:hep-th/9507143

9. H.F. Dowker, J.P. Gauntlett, G.W. Gibbons, G.T. Horowitz, Nucleation of $P$-branes and fundamental strings. Phys. Rev. D 53, 7115 (1996). arXiv:hep-th/9512154

10. D.V. Gal'tsov, O.A. Rytchkov, Generating branes via sigma models. Phys. Rev. D 58, 122001 (1998). arXiv:hep-th/9801180

\footnotetext{
${ }^{1}$ We remind the reader that an electric (magnetic) $p$-brane corresponds to a magnetic (electric) $F(D-3-p)$ fluxbrane; see [3] and the references therein.
} 
11. C.-M. Chen, D.V. Gal'tsov, S.A. Sharakin, Intersecting $M$-fluxbranes. Grav. Cosmol. 5(17), 45-48 (1999); arXiv:hep-th/9908132

12. M.S. Costa, M. Gutperle, The Kaluza-Klein Melvin solution in M-theory. JHEP 0103, 027 (2001). arXiv:hep-th/0012072

13. P.M. Saffin, Gravitating fluxbranes. Phys. Rev. D 64, 024014 (2001). arXiv:gr-qc/0104014

14. M. Gutperle, A. Strominger, Fluxbranes in string theory. JHEP 0106, 035 (2001). arXiv:hep-th/0104136

15. M.S. Costa, C.A. Herdeiro, L. Cornalba, Flux-branes and the dielectric effect in string theory. Nucl. Phys. B 619(1), 155-190 (2001). arXiv:hep-th/0105023

16. R. Emparan, Tubular branes in fluxbranes. Nucl. Phys. B 610, 169 (2001). arXiv:hep-th/0105062

17. P.M. Saffin, Fluxbranes from p-branes. Phys. Rev. D 64, 104008 (2001). arXiv:hep-th/0105220

18. J.M. Figueroa-O'Farrill, G. Papadopoulos, Homogeneous fluxes, branes and a maximally supersymmetric solution of $M$-theory. JHEP 0106, 036 (2001). arXiv:hep-th/0105308

19. D. Brecher, P.M. Saffin, A note on the supergravity description of dielectric branes. Nucl. Phys. B 613, 218 (2001). arXiv:hep-th/0106206

20. J.G. Russo, A.A. Tseytlin, Supersymmetric fluxbrane intersections and closed string tachyons. JHEP 11, 065 (2001). arXiv:hep-th/0110107

21. C.M. Chen, D.V. Gal'tsov, P.M. Saffin, Supergravity fluxbranes in various dimensions. Phys. Rev. D 65, 084004 (2002). arXiv:hep-th/0110164

22. J. Figueroa-O'Farrill and J. Simon, Generalized supersymmetric fluxbranes, JHEP 12, 011 (2001). arXiv:hep-th/0110170

23. R. Empharan, M. Gutperler, From p-branes to fluxbranes and back. JHEP 0112, 023 (2001). arXiv:hep-th/0111177

24. V.D. Ivashchuk, V.N. Melnikov, Multidimensional gravitational models: Fluxbrane and S-brane solutions with polynomials. AIP Conf. Proc. 910, 411-422 (2007)

25. I.S. Goncharenko, V. D. Ivashchuk, V.N. Melnikov, Fluxbrane and S-brane solutions with polynomials related to rank-2 Lie algebras, Grav. Cosmol. 13(52), 262-266 (2007); arXiv:math-ph/0612079

26. B. Kleihaus, J. Kunz, E. Radu, Nonabelian solutions in a Melvin magnetic universe. Phys. Lett. B 660, 386-391 (2008)

27. A.A. Golubtsova, V.D. Ivashchuk, Fluxbrane and S-brane solutions related to Lie algebras. Phys. Part. Nucl. 43(5), 720-722 (2012)
28. V.D. Ivashchuk, V.N. Melnikov, Multidimensional gravity, flux and black brane solutions governed by polynomials. Grav. Cosmol. 20(3), 182-189 (2014)

29. J. Fuchs, C. Schweigert, Symmetries, Lie algebras and representations. A graduate course for physicists (Cambridge University Press, Cambridge, 1997)

30. B. Kostant, Adv. in Math. 34, 195 (1979)

31. M.A. Olshanetsky, A.M. Perelomov, Invent. Math. 54, 261 (1979)

32. V.D. Ivashchuk, Black brane solutions governed by fluxbrane polynomials. J. Geom. Phys. 86, 101-111 (2014)

33. A.A. Golubtsova, V.D. Ivashchuk, On calculation of fluxbrane polynomials corresponding to classical series of Lie algebras; arXiv:0804.0757 [nlin.SI]

34. S.V. Bolokhov, V.D. Ivashchuk, On generalized Melvin solution for the Lie algebra $E_{6}$, arXiv:1706.06621

35. V.D. Ivashchuk, V.N. Melnikov, Multidimensional classical and quantum cosmology with intersecting $p$-Branes. J. Math. Phys. 39, 2866-2889 (1998). arXiv:hep-th/9708157

36. V.D. Ivashchuk, V.N. Melnikov, Exact solutions in multidimensional gravity with antisymmetric forms. Class. Quantum Gravity 18, R82-R157 (2001). arXiv:hep-th/0110274

37. V.D. Ivashchuk, V.N. Melnikov, On brane solutions related to non-singular Kac-Moody algebras, SIGMA 5, 070, (2009): arXiv:0810.0196

38. V.D. Ivashchuk, Flux integrals for fluxbrane solutions governed by polynomials (in preparation)

39. V.G. Kac, Infinite-dimensional Lie Algebras (Cambridge University Press, Cambridge, 1990)

40. M. Henneaux, D. Persson, P. Spindel, Spacelike singularities and hidden symmetries of gravity. Living Rev. Relativ. 11, 1-228 (2008)

41. M.E. Abishev, K.A. Boshkayev, V. D. Ivashchuk, Dilatonic dyonlike black hole solutions in the model with two Abelian gauge fields. Eur. Phys. J. C 77, 180 (2017) 